\title{
A neonatal rat model of increased right ventricular afterload by pulmonary artery banding
}

\author{
Shoubao Wang, MD, PhD, ${ }^{\text {a }}$ Lincai Ye, $\mathrm{PhD},{ }^{\mathrm{a}, \mathrm{b}, \mathrm{c}}$ Haifa Hong, MD, PhD, ${ }^{\mathrm{a}}$ Chao Tang, PhD, \\ Minghui Li, MD, PhD, ${ }^{a}$ Zhen Zhang, $\mathrm{MD}, \mathrm{PhD},{ }^{\text {b,c }}$ and Jinfen Liu, $\mathrm{MD}^{\mathrm{c}}$
}

\section{ABSTRACT}

Objective: To construct a neonatal rat model of increased right ventricular (RV) afterload for studying the pathophysiological remodeling of the right ventricle in patients with congenital heart disease with increased RV afterload.

Methods: Surgery was performed within 6 hours after birth. Horizontal thoracotomy was performed by dissecting the intercostal muscles and splitting the sternum. The PA was then banded with 11-0 nylon thread. At postnatal day 7 (P7), constriction of PA was confirmed by echocardiography. The RV systolic and diastolic pressures were measured by cardiac catheterization. The RV end-systolic volume, end-diastolic volume, end-diastolic diameter, and free wall thickness were assessed by magnetic resonance imaging. The histological changes in sham-operated and PA-banding (PAB) hearts were evaluated by hematoxylin and eosin staining.

Results: Increased RV afterload was established by constriction of the PA in neonatal rats within 6 hours after birth. The survival rate was $75 \%$ at P7. Relative to the sham group, the peak pressure gradient across the PA constriction and RV systolic and diastolic pressures, end-systolic volume, end-diastolic volume, enddiastolic diameter, and free wall thickness were significantly increased in the PAB group at $\mathrm{P} 7(P<.01)$. Consistently, histological examination showed that the RV free wall was significantly hypertrophic in the PAB group.

Conclusions: We successfully established a neonatal RV afterload increase model through PAB within 6 hours after birth, which can be used to study the pathophysiological changes in congenital heart diseases with increased RV afterload. ( $\mathbf{J}$ Thorac Cardiovasc Surg 2017;154:1734-9)

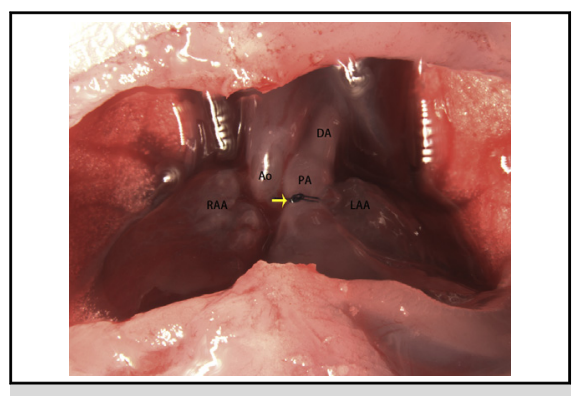

Constriction of the pulmonary artery in rats within 6 hours after birth.

Central Message
A neonatal increased right ventricular afterload
model was established by banding the pulmo-
nary artery within 6 hours after birth.

\section{Perspective}

Immature myocardium is different from mature myocardium in many aspects. However, our current understanding of neonatal right ventricular (RV) remodeling relies on knowledge gained from adult models. Here we show that it is feasible to generate a neonatal increased $\mathrm{RV}$ afterload rat model by banding the pulmonary artery within 6 hours after birth. We believe that this new model will greatly facilitate neonatal RV remodeling research.

See Editorial Commentary page 1740.

See Editorial page 1733.
Increased right ventricular (RV) afterload and subsequent dysfunction are often encountered in patients with 3 types of congenital heart disease: pulmonary artery (PA) hypertension associated with left-to-right shunt, RV outflow

From the ${ }^{\mathrm{a}}$ Department of Thoracic and Cardiovascular Surgery, ${ }^{\mathrm{b}}$ Institute of Pediatric Translational Medicine, and ${ }^{\mathrm{c}}$ Shanghai Pediatric Congenital Heart Disease Institute, Shanghai Children's Medical Center, Shanghai Jiaotong University School of Medicine, Shanghai, People's Republic of China.

S.W. and L.Y. contributed equally to this work.

This work was supported by Grants 2013CB945302 and 2013CB945304 from the National Basic Research Program of China, Grants 31371465 and 81170153 from the National Natural Science Foundation of China, Grant XBR2013105 from the Shanghai Health and Family Planning Commission, and the Program for Professor of Special Appointment (Eastern Scholar). obstruction (as in, eg, tetralogy of Fallot), and systemic $\mathrm{RV}$. Increased RV afterload initially results in compensated or adaptive hypertrophy, but persistent abnormal afterload will lead to decompensated or maladaptive RV hypertrophy

Received for publication March 24, 2017; revisions received May 9, 2017; accepted for publication June 1, 2017; available ahead of print July 8, 2017.

Address for reprints: Zhen Zhang, MD, PhD or Jinfen Liu, MD, Shanghai Pediatric Congenital Heart Disease Institute, Shanghai Children's Medical Center, Shanghai Jiao Tong University School of Medicine, 1678 Dong Fang Rd, Shanghai 200127, People's Republic of China (E-mail: zhenzhang@sjtu.edu.cn or liujinfen2014@ 163.com).

$0022-5223 / \$ 36.00$

Copyright () 2017 by The American Association for Thoracic Surgery

http://dx.doi.org/10.1016/j.jtcvs.2017.06.016 

Abbreviations and Acronyms
$\mathrm{H} \& \mathrm{E}=$ hematoxylin and eosin
LVWT $=$ left ventricular free wall thickness
MRI = magnetic resonance imaging
$\mathrm{P} \quad=$ postnatal day
$\mathrm{PA}=$ pulmonary artery
$\mathrm{PAB}=$ pulmonary artery banding
PPG $=$ peak pressure gradient
$\mathrm{RV} \quad=$ right ventricular
$\mathrm{RVP}=$ right ventricular pressure
RVWT $=$ right ventricular free wall thickness

Scanning this QR code will take you to a supplemental video for the article.

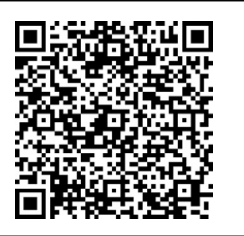

and then RV dysfunction, a condition in which the right ventricle is symptomatically unable to fill or eject properly. Although RV remodeling under increased afterload has been studied extensively in adults, ${ }^{1-4}$ the mechanisms of RV hypertrophy and dysfunction in childhood are poorly understood owing to the lack of an appropriate model.

It is well known that immature and mature myocardium differ in quite a few biochemical and physiological aspects. ${ }^{5}$ For example, immature myocardium is more dependent on glycolysis for ATP production due to abundant intracellular glycogen granules and more reliant on extracellular calcium for excitation-contraction coupling and intracellular calcium homeostasis. $^{6-9}$ Several recent studies have shown that mammalian heart can regenerate through cardiomyocyte proliferation within 1 week after birth in response to physical or ischemic lesions. ${ }^{10,11}$ Given all these different biological characteristics of immature myocardium, the knowledge gained from adult models might not completely comply with the remodeling of immature myocardium. Therefore, a reliable model that can recapitulate the pathophysiological status of increased RV afterload from the beginning of postnatal life is desirable.

Current increased RV afterload models are established either by hypoxia/monocrotaline-induced pulmonary hypertension or by PA trunk constriction in young large animals. ${ }^{12-16}$ Neither of these methods produces increased $\mathrm{RV}$ afterload immediately after birth, however. The former takes weeks to develop pulmonary hypertension, and the latter has never been reported in postnatal day 0 (P0) large animals. Even if PA banding (PAB) could be performed in P0 large animals, the cost and time for detailed molecular studies would make it a daunting task.
Recently, several common cardiac surgeries, including coronary artery ligation and apical resection, were successfully performed in neonatal rodents. ${ }^{10,11}$ Thus, we wonder whether the more complicated $\mathrm{PAB}$ procedure could be implemented in neonatal rodents.

In this study, we generated the first neonatal increased RV afterload rat model by constricting the PA within 6 hours after birth. Increased RV afterload and myocardial hypertrophy were confirmed by echocardiography, hemodynamic measurements, magnetic resonance imaging (MRI), and histology at P7. In doing so, we successfully simulated the pathophysiological status of increased RV afterload on immature myocardium, and the powerful genetic manipulation techniques available for rodents will further facilitate the molecular studies in RV remodeling.

\section{MATERIALS AND METHODS \\ Animals}

The surgical protocols followed in this investigation were approved by the Animal Care and Use Committee of Shanghai Children's Medical Center. PAB and sham operations were performed on neonatal SpragueDawley rats within 6 hours after birth and in adherence with the guidelines from the Committee for the Care and Use of Laboratory Animals.

Pregnant Sprague-Dawley rats were purchased from Xipu'er-bikai Experimental Animal Co, Ltd (Shanghai, China). At 6 hours after birth, the rat neonates (both males and females) were randomized to 2 groups: the experimental group (PAB group), comprising 30 neonatal rats that underwent PAB surgery, and the control group (sham group), comprising 30 neonatal rats that underwent same procedure except for the banding step.

Postoperative measurements, such as echocardiography studies, MRI evaluation, and histology, were conducted blindly. Twelve rats ( 6 for the

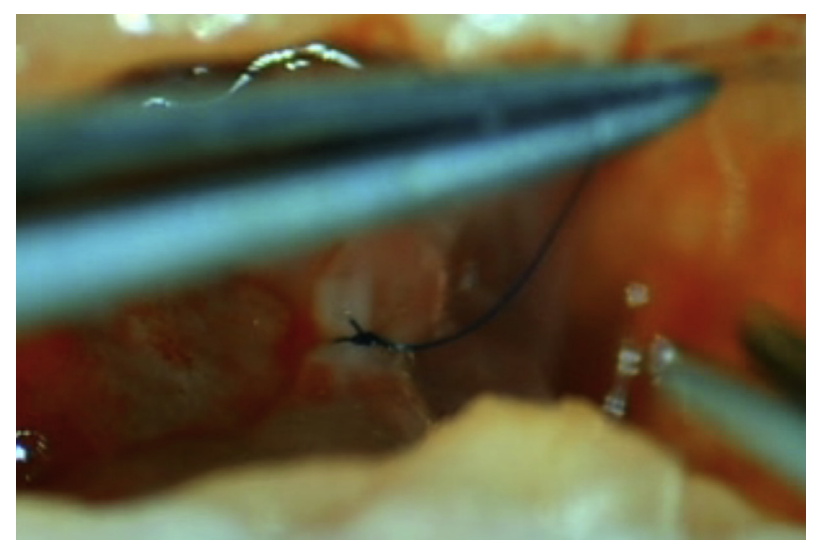

VIDEO 1. Constriction of the PA in a 4-hour-old rat. Following a transverse skin incision, horizontal thoracotomy at the third intercostal space was performed by dissecting intercostal muscles and cutting the sternum. After the pericardium was opened, the PA was separated from the aorta with micro scissors. A 11-0 nylon thread was positioned under pulmonary artery and a 30-Gauge needle was placed upon the pulmonary artery. The pulmonary artery and needle were then tied together. When the needle was removed, a fixed constricted opening was created. The sternum and thoracic wall were sutured with a 9-0 nylon thread. Afterward, the neonates were placed under a heat plate to warm up. Finally, the natural movements and a red/pink complexion were achieved. Video available at: http://www.jtcvsonline.org/ article/S0022-5223(17)31192-3/addons. 
sham group and 6 for the PAB group) were used first for echocardiography studies and then for hemodynamic measurements. Afterward, hearts were rapidly excised for histological analysis. The other 12 rats $(6$ in the sham group and 6 in the PAB group) were used for MRI evaluation. The remaining 36 rats were used for analyses presented elsewhere.

\section{Surgical Protocol}

PAB surgery was performed on the neonatal rats within 6 hours after birth (Video 1). Neonates were anesthetized by an approximately 4 minutes of direct cooling, which led to asystole and reversible apnea and prevented excessive blood loss during surgery. ${ }^{17}$ They were then transferred to an ice bed and fixed in the supine position under a stereomicroscope. Following a transverse skin incision, a horizontal thoracotomy at the third intercostal space was performed by dissecting the intercostal muscles and cutting the sternum. After the pericardium was opened along the main PA, the PA was carefully separated from the aorta under a stereomicroscope with microsurgical scissors. A 11-0 nylon thread was positioned under the PA, and a 30 -gauge needle $(0.31 \mathrm{~mm}$ diameter $)$ was placed on the PA. The PA and needle were then tied together by the thread. When the needle was removed, a fixed constricted opening equal to the diameter of the needle was created in the PA lumen. The sternum and thoracic wall were sutured with a 9-0 nylon thread. Afterward, the neonates were removed from the ice bed and placed under a $37^{\circ} \mathrm{C}$ heat plate to warm for several minutes until natural movements and a red/pink complexion were achieved. The neonates were then returned to their mothers. The entire procedure lasted approximately 17 minutes. The sham group underwent the same procedure except for the banding step.

\section{Transthoracic Echocardiography}

To confirm PA stenosis, transthoracic echocardiography was performed at 7 days after the surgery. Rats were anesthetized with isoflurane gas in a chamber (isoflurane/oxygen: $5 \%$ induction) and placed in a supine position. The rats were allowed to breathe spontaneously through a nasal cone (isoflurane/oxygen: $1.5 \%-2.0 \%$ maintenance). Echocardiograms were analyzed with a Vevo2100 imaging system (Visual Sonics, Toronto, Ontario, Canada) equipped with a 25-MHz transducer (MS400 MicroScan transducer; Visual Sonics). In addition, a long-axis view of the PA was used to measure the peak pressure gradient (PPG) across the PA constriction by continuous wave Doppler. All measurements were performed by an experienced ultrasound technician and recorded as the average of 3 consecutive cardiac cycles.

\section{Hemodynamic Measurements}

After transthoracic echocardiography, hemodynamic assessment of the right ventricle was performed by cardiac catheterization. In brief, neonates were anesthetized by inhalation of isoflurane ( $5 \%$ induction, $2 \%-3 \%$ maintenance), intubated, ventilated, and placed in the supine position. After a median sternotomy and pericardiotomy, the right ventricle was punctured with a 27 -gauge needle. A 4.5 -mm conductance catheter (Millar Instruments, Houston, TX) was inserted into the hole and positioned in the outflow tract to measure RV pressures. Data were recorded using a Power Lab system (AD Instruments, Colorado Springs, $\mathrm{CO}$ ) and analyzed using LabChart 7.0 software (AD Instruments).

\section{Histology}

Hearts were harvested and fixed in $4 \%$ paraformaldehyde overnight at room temperature, then dehydrated in ethanol series, embedded in paraffin, and sectioned into 6- $\mu \mathrm{m}$ slices. Hematoxylin and eosin (H\&E) staining was performed according to the standard procedure for routine light microscopy examination.

\section{Cardiac MRI}

Cardiac MRI was performed with a 7-T Bruker Biospec 7T/20 cm scanner (Bruker, Ettlingen, Germany) equipped with a $300-\mathrm{mT} / \mathrm{m}$ gradient

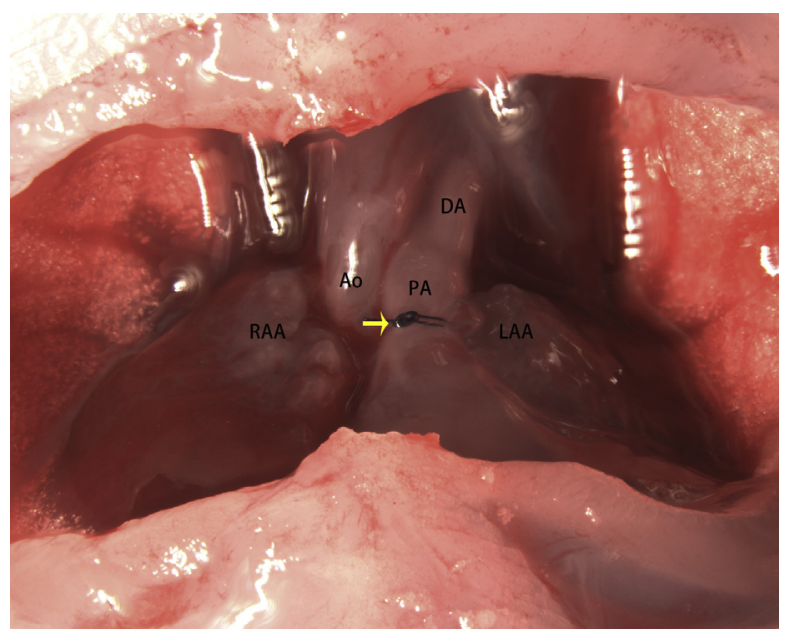

FIGURE 1. Illustration of the surgical technique for PAB (arrow). RAA, Right atrial appendage; $A o$, aorta; $P A$, pulmonary artery; $D A$, ductus arteriosus; $L A A$, left atrial appendage.

system and IntraGate self-gating tool (Bruker). Neonatal rats were anaesthetized by isoflurane ( $5 \%$ induction, $1.5 \%-2.0 \%$ maintenance) and respiratory rate was monitored continuously. The following imaging parameters were applied in image acquisition: field of view, $2.50 \times 2.50 \mathrm{~cm}$; repetition time, $10.0 \mathrm{~ms}$; echo time, $1.6 \mathrm{~ms}$; matrix, $128 \times 128$; slice thickness, $1.0 \mathrm{~mm}$; repetitions, 8 ; resolution, $0.0218 \mathrm{~mm} /$ pixel. All images were analyzed in OsiriX (DICOM viewer, version 3.5; Pixmeo, Geneva, Switzerland). The cardiac plane was localized by scout images detecting the 2- and 4-chamber views of the heart. Next, RV end-diastolic and RV
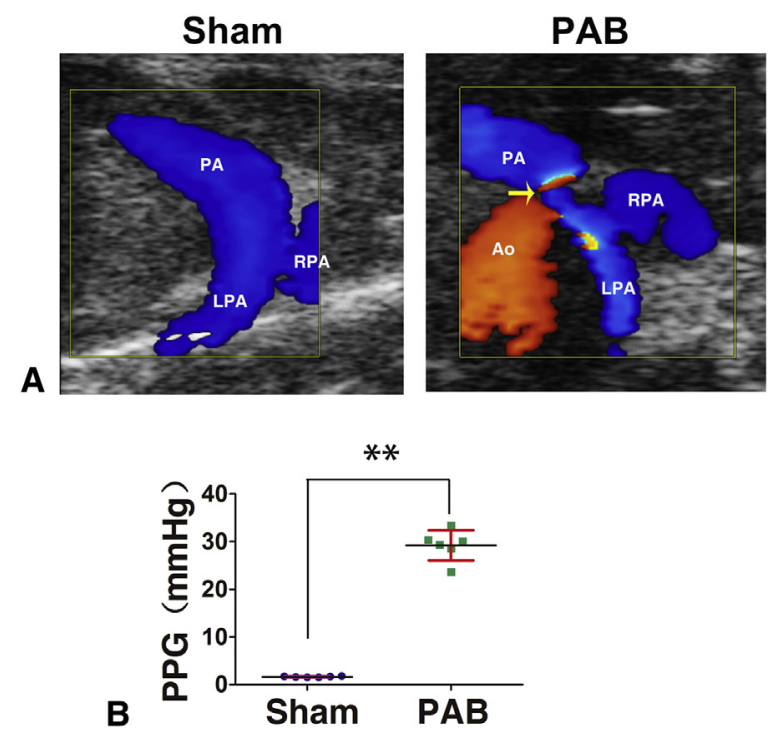

FIGURE 2. Echocardiographic images at $P 7$ after sham and PAB surgery in neonatal rats. A, The PA was detectable by echocardiography. The yellow arrow indicates the constriction site in the PA. B, Graphical comparison of the peak pressure gradient across PA constriction measured by transthoracic echocardiography between the sham and PAB groups. Data are presented as mean $\pm \mathrm{SD}$. Statistic analysis was done using the 2-tailed Student's $t$ test. $* * P<.01$. The power value was 0.99 with $\alpha$ set at 0.05 . $P A B$, Pulmonary artery banding; $P A$, pulmonary artery; $L P A$, left pulmonary artery; $R P A$, right pulmonary artery; $A o$, aorta; $P P G$, peak pressure gradient. 
TABLE 1. Hemodynamic status of RV at P7

\begin{tabular}{ccc}
\hline \multicolumn{1}{c}{ Parameter } & Sham Group $(\mathbf{n}=\mathbf{6})$ & PAB Group $(\mathbf{n}=\mathbf{6})$ \\
\hline $\mathrm{RVP}_{\text {systolic }}, \mathrm{mmHg}$ & $8.25 \pm 0.61$ & $33.25 \pm 3.04 *$ \\
$\mathrm{RVP}_{\text {diastolic }}, \mathrm{mmHg}$ & $2.77 \pm 0.45$ & $6.64 \pm 0.79 *$ \\
\hline
\end{tabular}

All data are mean \pm SD. Statistical analyses were done using the 2-tailed Student $t$ test ( 6 for the sham group and 6 for the PAB group). $P<.05$ is considered significant. Both $\mathrm{RVP}_{\text {systolic }}$ and $\mathrm{RVP}_{\text {diastolic }}$ have a power value of 0.99 with $\alpha$ set at 0.05. PAB, Pulmonary artery banding; $R V P$, right ventricular pressure. ${ }^{*} P<.01$

end-systolic images were acquired with a multislice short-axis view of the right ventricle. Six contiguous axial slices were acquired for complete coverage of the left and right ventricles. The midpoint of the right ventricle on the fourth slice (starting from the apex) was used to measure RV free wall thickness (RVWT), RV end-diastolic diameter, left ventricular free wall thickness (LVWT), and septum thickness. RV volume was calculated by measuring all the contiguous axial slices of the RV chamber.

\section{Quantification and Statistical Analysis}

Quantitative data are presented as mean \pm SD. Statistical analyses were performed with the 2-tailed Student's $t$ test for normally distributed groups. $P<.05$ was considered to indicate significance. Power calculations were performed to confirm statistical significance, with $\alpha$ set at 0.05 .

\section{RESULTS}

\section{Survival}

To study the neonatal RV remodeling in response to increased afterload, we performed PAB within 6 hours of birth. Under hypothermia anesthesia, the main PA was irreversibly constricted to a diameter of $0.31 \mathrm{~mm}$ with the nylon thread (Figure 1). The survival rate after surgery was approximately $92 \% ; 5$ pups ( 3 in the PAB group and 2 in the sham group) were unable to wake up after surgery.
Ten rats (6 in the PAB group, 4 in the Sham group) died of maternal cannibalization over the next 2 days, and 45 rats remained alive at $\mathrm{P} 7$; therefore, the survival rate was reduced to $75 \%$ at $\mathrm{P} 7$. Lethality was higher in the $\mathrm{PAB}$ pups compared with the sham pups.

\section{Echocardiographic Study}

PA constriction could be detected in rats that had undergone PAB surgery (Figure 2, A, arrow). Irregular color patterns observed at the constriction site and poststenosis area indicate stenosis-induced fast turbulent flow (Figure 2, A). A long-axis view of the PA was used to measure the PPG across the PA constriction by continuous wave Doppler. An average 18-fold increase in the PPG across the PA constriction site was noted in the PAB group compared with the sham group at P7 (Figure 2, B). Specifically, at P7, the PPG across the PA constriction was $29.21 \pm 3.17 \mathrm{mmHg}$ in the $\mathrm{PAB}$ group, but only $1.63 \pm 0.11 \mathrm{mmHg}$ in the sham group (Figure 2, B), indicating significantly increased RV afterload following $\mathrm{PAB}$ surgery.

\section{Hemodynamic Study}

Hemodynamic assessment of RV was performed by cardiac catheterization. The results showed an average 4-fold increase in $\mathrm{RV}$ systolic pressure $\left(\mathrm{RVP}_{\text {systolic }}\right)$ in the $\mathrm{PAB}$ group at $\mathrm{P} 7$ (Table 1). Specifically, mean $\mathrm{RVP}_{\text {systolic }}$ in the $\mathrm{PAB}$ group at $\mathrm{P} 7$ was $33.25 \pm 3.04 \mathrm{mmHg}$, significantly higher than the $8.25 \pm 0.61 \mathrm{mmHg}$ in the sham group (Table 1). Consistently, mean RV diastolic pressure
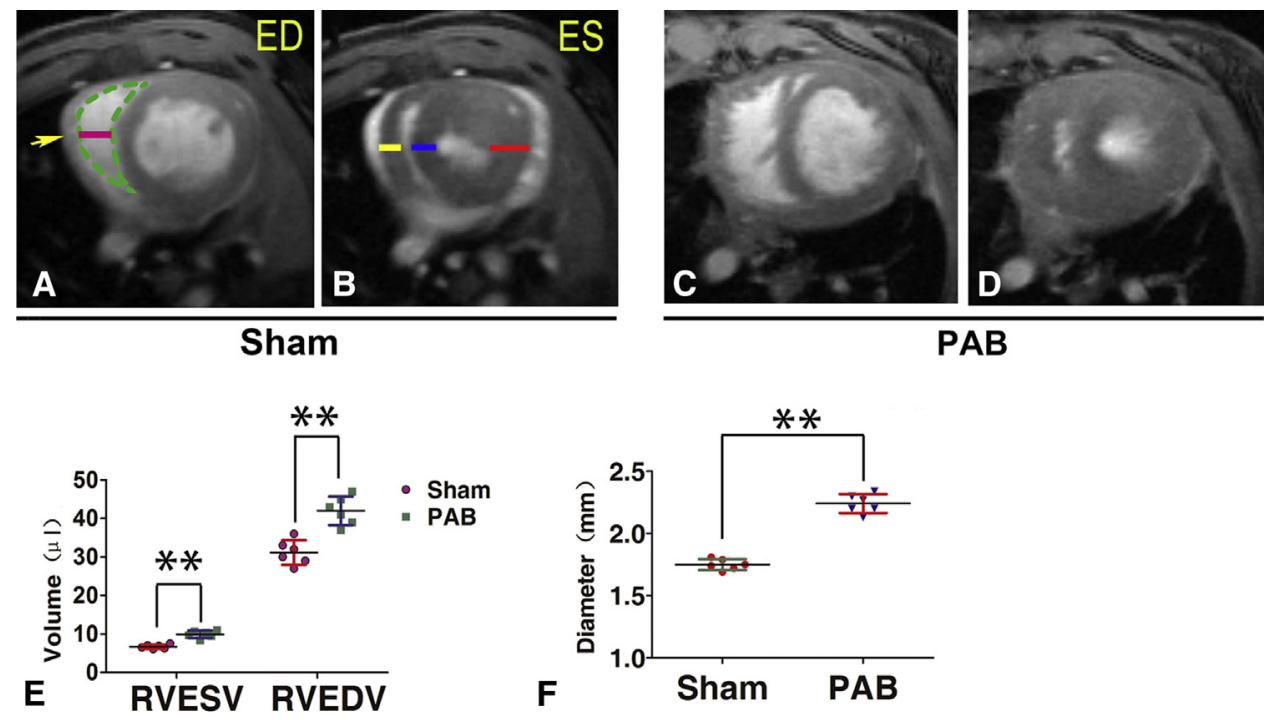

FIGURE 3. Morphological changes of heart at postnatal day 7 after the sham and PAB surgery in neonatal rats. A-D, Representative MRI images. A and C, $\mathrm{ED}$, end of diastole. B and D, ES, end of systole. The arrow denotes the right ventricle; yellow line, RVWT; blue line, septum thickness; red line, LVWT; purple line, RV end-diastolic diameter (RVEDD); dashed line, RV chamber. E, Quantitative comparison of RV end-diastolic volume (RVEDV) and RV endsystolic volume (RVESV) between the sham and PAB groups. F, Quantitative comparison of RVEDD between the sham and PAB groups. Data are presented as mean $\pm \mathrm{SD}$ ( 6 for the sham group and 6 for the PAB group). Statistical analysis was done with the 2-tailed Student's $\mathrm{t}$ test. $* * P<.01$. The power values for RVEDV, RVESV, and RVEDD were 0.99 with $\alpha$ set at 0.05 . PAB, Pulmonary artery banding; $E D$, end of diastole; $E S$, end of systole. 
TABLE 2. Quantification of RV Hypertrophy at P7

\begin{tabular}{lcc}
\hline \multicolumn{1}{c}{ Parameter } & Sham Group $(\mathbf{n}=\mathbf{6})$ & PAB Group $(\mathbf{n}=\mathbf{6})$ \\
\hline RVWT/LVWT & $0.4763 \pm 0.0105$ & $0.6238 \pm 0.0174^{*}$ \\
RVWT/septum thickness & $0.9642 \pm 0.0219$ & $1.258 \pm 0.0374^{*}$ \\
LVWT/septum thickness & $2.025 \pm 0.0169$ & $2.0165 \pm 0.0189$ \\
\hline
\end{tabular}

All data are presented as mean \pm SD. Statistical analyses were done using the 2-tailed Student's $t$ test ( 6 for the sham group and 6 for the PAB group). $P<.05$ is considered significant. The power values of ratios of RVWT/LVWT, RVWT/septum thickness, and LVWT/septum thickness were $0.99,0.99$ and 0.88 , respectively, with $\alpha$ set at 0.05 . $P A B$, Pulmonary artery banding; $R V W T$, right ventricular free wall thickness; $L V W T$, left ventricular free wall thickness. $* P<.01$.

$\left(\mathrm{RVP}_{\text {diastolic }}\right)$ was $6.64 \pm 0.79 \mathrm{mmHg}$ in the $\mathrm{PAB}$ group, compared with only $2.77 \pm 0.45 \mathrm{mmHg}$ in the sham group (Table 1). These results indicate the successful establishment of increased RV afterload and subsequent $\mathrm{RV}$ pressure increase as early as $\mathrm{P} 7$ after PAB in neonates.

\section{Cardiac MRI Results}

To evaluate the effect of the PAB surgery on RV structure and function, we used MRI to visualize the right ventricle at P7. In contrast to the sham group, the PAB rats exhibited dramatic increases in RV end-systolic volume, RV enddiastolic volume, and RV end-diastolic diameter, indicating significant RV dilation after the PAB surgery (Figure 3, $A-F)$. To accurately evaluate the degree of RV hypertrophy in response to PAB, we normalized RVWT to LVWT or septum thickness. The results show significantly increases in RVWT/LVWT and RVWT/septum thickness ratios in

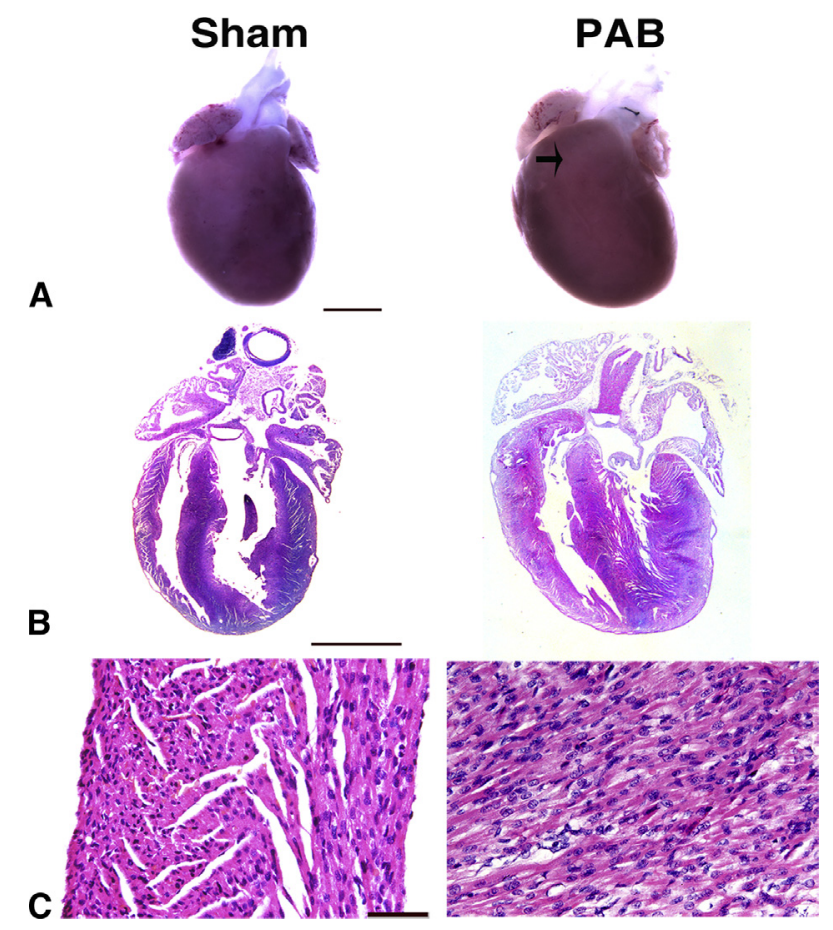

FIGURE 4. A, Representative view of whole heart at P7. (Scale bar: $2 \mathrm{~mm}$.) B, H\&E-stained 4-chamber coronal sections of a P7 heart. (Scale bar: $2 \mathrm{~mm}$.) C, High magnification of H\&E-stained sections of P7 right ventricle. (Scale bar: $50 \mu \mathrm{m}$.) $P A B$, Pulmonary artery banding. neonatal $\mathrm{PAB}$ rats at $\mathrm{P} 7$ compared with the sham rats, but not in LVWT/septum thickness ratio (Table 2). Overall, PAB could cause significant RV dilation and hypertrophy as early as 7 days after surgery.

\section{Histological Examination of Hearts}

To clarify the histological changes of the sham and PAB hearts, we performed $\mathrm{H} \& \mathrm{E}$ staining for microscopic examination. In a gross view, the PAB heart was significantly enlarged at P7 (Figure 4, $A$ ), particularly in the infundibulum portion of the right ventricle under the PA (Figure 4, A, arrow). Consistent with MRI results and the gross view, we also observed a thickened RV free wall in the PAB heart (Figure 4, B). Furthermore, in the highermagnification H\&E-stained sections, we noted slightly disorganized myocardial fibers, enlarged cardiomyocytes, and enlarged nuclei in the PAB heart (Figure 4, C), indicating cellular remodeling in the neonatal PAB heart.

\section{DISCUSSION}

The lack of a neonatal RV overload model has hindered our understanding of how neonatal RV reacts to the pathophysiological conditions in numerous congenital heart diseases. Here, with current neonatal surgery techniques, we successfully established a neonatal increased RV afterload model by banding the PA within 6 hours after birth. Different from current PAB models, the new model induces an acute RV afterload increase immediately after birth; therefore, it can be used to study the remodeling mechanism of neonatal myocardium. Compared with the adult PAB model, the progress of RV remodeling in neonatal $\mathrm{PAB}$ rats is much faster. All of the surviving PAB rats at P7 developed significant RV hypertrophy, whereas it usually takes 2 weeks to observe significant RV hypertrophy in adult PAB models. ${ }^{18,19}$ The extent of RV hemodynamic changes also was more dramatic in neonatal PAB rats. Compared with the approximate 2-fold increase in adult PAB rats, ${ }^{18,19}$ an average 4-fold increase of $\mathrm{RVP}_{\text {systolic }}$ is noted in neonatal $\mathrm{PAB}$ rats. Interestingly, although the neonatal $\mathrm{PAB}$ rates had a greater fold change in $\mathrm{RVP}_{\text {systolic, }}$ they had less change in end-diastolic volume. Specifically, neonatal PAB rats had an average 1.35-fold increase in end-diastolic volume, compared with the average 2 -fold increase in end-diastolic volume noted in adult PAB rats. ${ }^{18}$ All of these discrepancies suggest that different cellular and molecular mechanisms function in neonatal RV remodeling, which will require further molecular studies with this newly established model.

In this study, the survival rate at $\mathrm{P} 7$ was $70 \%$ for the PAB group and $80 \%$ for the sham group. This indicates that neonatal rats are quite tolerant of the PAB procedures, although it takes significantly longer than apical resection procedures (approximately 17 minutes vs $10 \mathrm{mi}-$ nutes). Extra care should be applied during PA dissection 
to avoid damaging the great arteries, which are quite fragile at the neonatal stage. For this reason, we chose to perform this step under a stereomicroscope. In addition, to provide a larger operating space, horizontal thoracotomy was performed by dissecting the unilateral intercostal space and also cutting the sternum along the intercostal space.

Some caveats in our studies must be mentioned, however. First, a fixed orifice constriction in P0 neonates severely constrains the pulmonary blood flow increase concomitant with rapid postnatal growth, which would limit the time range of changes before the neonate goes into circulatory decompensation due to severe constriction. Second, hemodynamic measurements were obtained under anesthesia and with the chest and pericardium open, both of which can alter pressure measurement values. ${ }^{20,21}$ Third, the PPG across the PA constriction site was measured by ultrasonography. Although the correlation of Doppler-estimated maximal gradient to peak-to-peak catheterization gradient is linear, studies have showed that Doppler measurement usually leads to a systematic overestimation of pressure drop. ${ }^{22}$ Fourth, postoperative analgesia was not applied.

\section{Conflict of Interest Statement}

Authors have nothing to disclose with regard to commercial support.

\section{References}

1. Voelkel NF, Quaife RA, Leinwand LA, Barst RJ, McGoon MD, Meldrum DR, et al. Right ventricular function and failure: report of a National Heart, Lung, and Blood Institute working group on cellular and molecular mechanisms of right heart failure. Circulation. 2006;114:1883-91.

2. Bartelds B, Borgdorff MA, Smit-van Oosten A, Takens J, Boersma B, Nederhoff MG, et al. Differential responses of the right ventricle to abnormal loading conditions in mice: pressure vs. volume load. Eur J Heart Fail. 2011; $13: 1275-82$.

3. Bogaard HJ, Natarajan R, Henderson SC, Long CS, Kraskauskas D, Smithson L, et al. Chronic pulmonary artery pressure elevation is insufficient to explain right heart failure. Circulation. 2009;120:1951-60.

4. Tang D, Yang C, del Nido PJ, Zuo H, Rathod RH, Huang X, et al. Mechanical stress is associated with right ventricular response to pulmonary valve replacement in patients with repaired tetralogy of Fallot. J Thorac Cardiovasc Surg. 2016;151:687-94.

5. Yamamoto F. Metabolic characteristics of immature myocardium. Gen Thorac Cardiovasc Surg. 2010;58:171-3.

6. Rolph TP, Jones CT. Regulation of glycolytic flux in the heart of the fetal guinea pig. J Dev Physiol. 1983;5:31-49.
7. Lopaschuk GD, Spafford MA, Marsh DR. Glycolysis is predominant source of myocardiol ATP production immediately after birth. Am J Physiol. 1991;261(6 Pt 2):H1698-705.

8. Grosso MA, Banerjee A, St Cyr JA, Rogers KB, Brown JM, Clarke DR, et al. Cardiac 5'-nucleotidase activity increases with age and inversely relates to recovery from ischemia. J Thorac Cardiovasc Surg. 1992;103:206-9.

9. Bolling SF, Olszanski DA, Bove EL, Childs KF. Enhanced myocardial protection during global ischemia with 5'-nucleotidase inhibitors. J Thorac Cardiovasc Surg. 1992;103:73-7.

10. Porrello ER, Mahmoud AI, Simpson E, Hill JA, Richardson JA, Olson EN, et al Transient regenerative potential of the neonatal mouse heart. Science. 2011;331: 1078-80.

11. Haubner BJ, Adamowicz-Brice M, Khadayate S, Tiefenthaler V, Metzler B, Aitman T, et al. Complete cardiac regeneration in a mouse model of myocardial infarction. Aging (Albany NY). 2012;4:966-77.

12. Tatebe S, Miyamura H, Sugawara M, Watanabe H, Eguchi S. Induction of righ ventricular hypertrophy in neonatal guinea pigs by monocrotaline. Jpn Circ J 1996;60:604-8.

13. Ambalavanan N, Nicola T, Hagood J, Bulger A, Serra R, Murphy-Ullrich J, et al Transforming growth factor-beta signaling mediates hypoxia-induced pulmonary arterial remodeling and inhibition of alveolar development in newborn mouse lung. Am J Physiol Lung Cell Mol Physiol. 2008;295:86-95.

14. Abduch MC, Assad RS, Rodriguez MQ, Valente AS, Andrade JL, Demarchi LM, et al. Reversible pulmonary trunk banding III: assessment of myocardial adaptive mechanisms-contribution of cell proliferation. J Thorac Cardiovasc Surg. 2007; 133:1510-6

15. Kitahori K, He H, Kawata M, Cowan DB, Friehs I, Del Nido PJ, et al. Development of left ventricular diastolic dysfunction with preservation of ejection fraction during progression of infant right ventricular hypertrophy. Circ Heart Fail. 2009;2:599-607.

16. Sheikh AM, Barrett C, Villamizar N, Alzate O, Valente AM, Herlong JR, et al Right ventricular hypertrophy with early dysfunction: a proteomics study in a neonatal model. J Thorac Cardiovasc Surg. 2009;137:1146-53.

17. Kulandavelu S, Qu D, Sunn N, Mu J, Rennie MY, Whiteley KJ, et al. Embryonic and neonatal phenotyping of genetically engineered mice. ILAR J. 2006;47: 103-17.

18. Kapur NK, Paruchuri V, Aronovitz MJ, Qiao X, Mackey EE, Daly GH, et al. Biventricular remodeling in murine models of right ventricular pressure overload. PLoS One. 2013;8:e70802.

19. Novoyatleva T, Schymura Y, Janssen W, Strobl F, Swiercz JM, Patra C, et al. Deletion of Fn14 receptor protects from right heart fibrosis and dysfunction. Basic Res Cardiol. 2013;108:325.

20. Kubitz JC, Annecke T, Kemming GI, Forkl S, Kronas N, Goetz AE, et al. The influence of positive end-expiratory pressure on stroke volume variation and central blood volume during open and closed chest conditions. Eur J Cardiothorac Surg. 2006;30:90-5.

21. Kawasaki T, Hoka S, Okamoto H, Takahashi S. The effect of the pericardium on right and left ventricular diastolic functions during isoflurane anesthesia. Anesth Analg. 1993;76:1195-200.

22. Yamashita T, Moriyama Y, Sata N, Hamada N, Horinouchi T, Amitani S, et al. Discrepancy between Doppler and catheter measurements of pressure gradients across small-size prosthetic valve. Jpn J Thorac Cardiovasc Surg. 2005;53:64-8.

Key Words: pulmonary artery banding, neonatal rat model, right ventricular, afterload 